\title{
The new normal - dentistry and the autistic patient
}

\author{
Wendy Bellis ${ }^{1}$
}

\section{Key points}

Oral care and dental treatment can be a challenge for many patients with autism spectrum disorder.
The COVID-19 pandemic and lockdown present even more barriers to health and care for many of our patients with autism.
As dental care resumes, dental professionals will need to ask patient and carers how the pandemic has affected, and still is affecting, them.

\begin{abstract}
This article looks at some of the issues around providing and receiving dental care for autistic children and adults in the shadow of COVID-19 and suggests management approaches. COVID -19 represents yet another barrier to accessing dental care for some individuals with ASD.Dental professionals need to specifically ask families at recall about how the pandemic has, and continues to affect the individual concerned. Dental management may consequently need to be adapted to reflect this information.
\end{abstract}

\section{Introduction}

The maintenance of oral health for the autistic patient can sometimes be a challenge for families and dental professionals

The repercussions caused by the COVID19 pandemic have been widely experienced by both the public and the dental profession, and the practice of dentistry has, of necessity, changed. These changes, together with lifestyle changes and lockdown, can particularly affect an individual with autism spectrum disorder (ASD).

Many of us are struggling to get used to a 'new normal', not only in our professional role but also in our everyday lives. For the patient with ASD, this struggle can be unbelievably distressing and in some cases almost impossible.

The barriers to dental care for the autistic individual and their families are well recognised and include issues around social communication difficulties, behavioural difficulties and sensory sensitivities. ${ }^{1}$

${ }^{1}$ Honorary Clinical Senior Teaching Fellow, Eastman Dental Institute, London UK.

Correspondence to: Wendy Bellis

Email address: wendybellis@nhs.net

Accepted 1 December 2020

https://doi.org/10.1038/s41415-021-3376-2
Comorbidities such as anxiety, learning difficulties, epilepsy and ADHD can also cause problems with oral health and receiving dental care.

\section{Problems for the patient}

Many people with ASD need to have routine and predictability in their lives, and often do not respond well to sudden changes. Disruption caused by restrictions and lockdown often cause anxiety and stress due to disturbances in familiar patterns and daily/weekly schedules at home, in schools and other settings. This can include changes in the people who they have learned to trust, such as caregivers, teachers, family members and care professionals.

Social media postings from members of the autistic communities suggest that isolation and lockdown can be a huge challenge for an individual and their carers. Normal support services (including respite) for families have also been limited severely in some areas, resulting in stress and heavy pressures on families.

Access to dental care has also been challenging, with recalls and regular care being halted or difficult to organise for many. The trusting relationships that had been built up over time with dental staff may have suffered. Access to emergency care may have been affected with pressures of general anaesthetic services, which are essential for certain people with ASD. Difficulty in expressing pain is not uncommon in patients with ASD and this is, understandably, a great worry for many parents and carers with limited access to dental services.

Some people with ASD require special diets or have strong dietary preferences, and accessing these may be compromised for several reasons including supply disruption, cost and travelling to shops. This can be extremely disturbing for these individuals, for whom getting used to a different brand, colour or consistency of food is not a realistic option.

Where a person with ASD has had to be hospitalised, the lack of a familiar person and visiting restrictions will have been especially difficult for them.

As dental staff, we are all familiar with wearing face masks. However, for a person with sensory issues, wearing a mask may be impossible. They may not be able to tolerate the feel or smell of the mask. Information is available on the National Autistic Society website, ${ }^{2}$ which is aimed at making mask wearing more acceptable for individuals. It is interesting that the advice mentions that the smell of a person's breath will be intensified by wearing a mask, and good oral hygiene and brushing teeth for wearing a mask is recommended. 


\section{Problems for dentists}

\section{New developments}

Child/patient and family functioning may have changed during COVID-19 and lockdown. For example, the usual caregivers may have changed, which in turn may impact on oral hygiene support. Medication may have been changed in both nature and dosage, and access to specific foods may also have been compromised.

\section{Action}

You may have to approach the child or adult almost as if they are a new patient. Ask the family how COVID-19 has affected them.

The British Society of Paediatric Dentistry (BSPD) pre-appointment questionnaire for children with autism could be used, ${ }^{3}$ together with any accessory questions to pick up any new developments or patterns of behaviour which might affect the patient's oral health and your dental care.

\section{Communication}

Mask wearing by dental staff can be a real barrier to communication, as many autistic people look more readily at another person's mouth rather than their eyes.

\section{Action}

Consider having a photo of yourself with and without a mask on the wall or waiting room door. The use of a transparent mask should be considered whenever possible to aid communication. If the dental staff have changed at the practice, then it would be wise to inform the child and family beforehand.

The BSPD website ${ }^{4}$ has material aimed at familiarising the neurotypical child to dental staff wearing masks. Although this could be used for children with autism, more specific and visual explanations may be required.
Many dentists are already familiar with the construction and use of social stories in their practice. Such visual aids can help an autistic individual prepare for the dental visit and reduce unpredictability. ${ }^{5}$

Mask wearing and any changes in the usual setup and running of the dental practice could be integrated into existing social stories, or you may have to construct a completely new one with the family. Next time you are irritated by a person in public who you think should be wearing a face mask, think twice.

\section{Clinical}

The time available for treating patients seems to have shrunk, whereas waiting lists seem to have grown.

Clinical challenges are many and varied; for example, there may have been changes in diet, oral hygiene and access to high-fluoride toothpaste, and an individual's disease risk status may also have changed. Also, the relationship which you and your staff had carefully built up with the patient may have suffered due to lack of exposure and reinforcement. It might now be difficult or take more time to encourage the individual to accept clinical treatment.

\section{Action}

Management of the autistic patient takes time. Bear in mind what has gone before, but also be aware of changes that might impact on your clinical care, particularly around sensory sensitivities and behaviours. Review and refresh preventive practices. Sensory processing in autistic children can be different and, together with communication barriers, the chid may not be able to express if they are in pain and/or locate the source accurately. Questions about changes in other spheres, such as in eating, sleeping and stimming (self-stimulatory behaviour), may help in the diagnosis of a problem. If possible, consider temporisation while this relationship of trust is rebuilt or if the child has to be placed on a waiting list for treatment under general anaesthesia. If the child is distressed and in an emergency situation, then it would be wise to be familiar with the 'Clinical Holding' document on the BSPD website ${ }^{6}$ before proceeding.

\section{Conclusion}

In conclusion, we are all facing the challenges posed by COVID-19, both in our professional and everyday lives. People with ASD are exposed to similar challenges, but may experience them in different and more profound ways. All members of the dental team will have to help them get used to our 'new normal.

\section{Ethics declarations}

The author declares no conflicts of interest.

\section{References}

1. Eades D, Leung P, Cronin A, Monteiro J, Johnson A, Remington A. UK dental professionals' knowledge, experience and confidence when treating patients on the autism spectrum. Br Dent J 2019; 227: 504-510.

2. National Autistic Society. Tips for autistic people and families. 2020. Available at https://www.autism.org. uk/advice-and-guidance/resources/tips-for-autisticpeople-and-families (accessed November 2020).

3. British Society of Paediatric Dentistry. Advice for parents of children with autism and sample questionnaire to accompany guide. 2020. Available online at https://www.bspd.co.uk/Portals/0/ Questionnaire $\% 20$ for $\% 20$ parents $\% 20$ of $\% 20$ children\%20with\%20autism\%20-\%20FINAL\%20 BSPD\%20Jan\%202017.docx (accessed November 2020).

4. British Society of Paediatric Dentistry. COVID-19 Dental Resources for Children. 2020. Available online at https://www.bspd.co.uk/Professionals/Resources/ COVID-19/COVID-19-Dental-Resources-for-Children (accessed November 2020).

5. Bellis W J. Will You See My Son? In Banerjee A Thavaraj $S$ (eds) Odell's Clinical Problem Solving in Dentistry. 4th ed. pp 231-235. China: Elsiever, 2020.

6. British Society of Paediatric Dentistry. A policy document on the use of Clinical Holding in the dental care of children. 2016. Available at https://www.bspd. co.uk/Portals/0/BSPD\%20clinical\%20holding\%20 guidelines $\% 20$ final\%20with $\% 20$ flow $\% 20$ chart $\% 20$ 250416.pdf (accessed November 2020). 